\title{
Perfil Cardiometabólico de Corredores de Rua Recreacionais
}

\author{
Cardiometabolic Profile of Recreational Street Runners
}

\author{
REABIAS DE ANDRADE PEREIRA ${ }^{1}$ \\ ORRANETTE PEREIRA PADILHAS ${ }^{2}$ \\ DOUGLAS CAVALCANTE SILVA ${ }^{3}$ \\ MATHEUS DA SILVEIRA COSTA ${ }^{4}$ \\ MARIA DE FÁTIMA IÊDA BARROSO DE OLIVEIRA ${ }^{3}$ \\ GUSTAVO DA SILVAFÉLIX ${ }^{5}$
}

\section{RESUMO}

Objetivo: Avaliar o perfil cardiometabólico de corredores recreacionais e comparar com os valores clínicos considerados limites para proteção cardiometabólica. Material e Métodos: Foram avaliados 99 atletas corredores recreacionais da cidade de João Pessoa, participantes regulares de competições, sendo 69 homens, idade média de 42,9 $\pm 9,6$ (homens) e 38,5 $\pm 8,4$ (mulheres). Após um jejum de 12 horas, foram realizadas coleta sanguínea para posterior análise do perfil lipídico, perfil glicêmico, atividades de enzimas hepáticas e função renal, além de medida de pressão arterial e avaliação antropométrica. Resultados: 0 marcador lipídico LDLc demonstrou-se acima dos valores de referência nos homens $(136,7 \pm 35,2$ vs $110 ; p=0,00)$, nas mulheres $(134,1 \pm 37,3$ vs $110 ; p=0,01)$ e em ambos os gêneros juntos $(130,6 \pm 36,3$ vs $110 ; p=0,00)$. A uréia estava com valores acima da normalidade $(53,8 \pm 16,6$ vs $45 ; p=0,00)$ apenas nos homens. Os outros marcadores de perfil glicêmico, funções hepática, renal e de pressão arterial encontraram-se dentro dos limites de normalidade. Conclusão: O perfil cardiometabólico de corredores recreacionais da cidade de João Pessoa encontra-se comprometido apenas do ponto de vista lipídico para homens e mulheres e renal apenas para os homens, tendo todos os outros parâmetros de saúde avaliados em perfeito estado. Considerando a idade dos corredores, este é um perfil mais protetor do que o da população em geral, o que parece indicar um efeito benéfico do treinamento de corrida.

\section{DESCRITORES}

Corrida. Corredores Recreacionais. Saúde. Cardiometabólico.

\begin{abstract}
Objective: To evaluate the cardiometabolic profile of recreational runners and compare to clinical values considered limits for cardiometabolic protection. Methods: Were evaluated 99 recreational runners athletes of the João Pessoa city, regular participants of competitions, 69 male, aged $42.9 \pm 9.6$ (male) and 38.5 \pm 8.4 (female). After a 12 hours fasting period, a blood collection for analysis of lipid and glycemic profile, hepatic enzyme activities and renal function was performed, together with blood pressure measurement and anthropometric assessment. Results: The LDLc lipid marker was above the reference values in males $(136.7 \pm 35.2$ vs $110 ; p=0.00)$ in female $(134.1 \pm 37.3$ vs $110 ; p=0.01)$ and in the genders together $(130.6 \pm 36.3 \mathrm{vs}$ $110 ; p=0.00)$. Urea was above normal values $(53.8 \pm 16.6$ vs $45 ; p=0.00$ ) only in male. The markers of glycemic profile, hepatic and renal function and blood pressure were within normal limits. Conclusion: Cardiometabolic profile of recreational runners athletes of the João Pessoa city is injured only, for male and female, in lipid perspective and renal only in male, with all other health parameters evaluated in perfect condition. Considering the age of the runners, this is a more protective profile than the general population, which seems to indicate a beneficial effect of the training running.
\end{abstract}

\section{DESCRIPTORS}

Running. Recreational Runners. Health. Cardiometabolic.

\footnotetext{
Graduado em Educação Física, Universidade Federal da Paraíba, João Pessoa-PB, Brasil

Mestre em Educação Física, Universidade Federal da Paraíba, João Pessoa-PB, Brasil

Graduando (a) em Educação Física, Universidade Federal da Paraíba, João Pessoa-PB, Brasil

4 Graduado em Nutrição, Universidade Maurício de Nassau, Campina Grande-PB, Brasil

5 Mestrando em Educação Física, Universidade Federal da Paraíba, João Pessoa-PB, Brasil
} 
$\mathrm{S}$ abe-se que o treinamento físico é associado com a proteção cardiovascular, além de ser uma ferramenta não medicamentosa eficaz no tratamento de enfermidades cardiometabólicas tais como hipertensão arterial, diabetes e obesidade ${ }^{1}$. É bem aceito que as modalidades esportivas praticadas com intensidade moderada são as mais eficazes para promover proteção cardiometabólica ${ }^{2}$. A prevalência de pessoas que praticam exercício de caráter moderado tem aumentando nos últimos anos. No Brasil, segundo dados do VIGITEL (Vigilância de Fatores de Risco e Proteção para Doenças Crônicas por Inquérito Telefônico), a prevalência de pessoas suficientemente ativas aumentou de 30 para $35 \%$ nos últimos seis anos ${ }^{3}$.

Paralelamente a esta mudança comportamental, outro interessante fenômeno tem surgido no Brasil e no mundo. Trata-se de um aumento percentualmente bem maior de pessoas que estão aderindo à prática da corrida de rua de forma amadora. No Brasil, estima-se que aproximadamente $5 \%$ da população aderiram à corrida de rua como prática esportiva, o que equivale a cerca de 10 milhões de corredores ${ }^{4}$. Esse fato é verificado em corridas como a São Silvestre, que teve 30.000 participantes em sua última edição (2014), enquanto nos cinco anos anteriores o número ficou em torno de 25 mil, assim como a Maratona Internacional de São Paulo que em 2015 teve 19 mil inscritos, e em 2008 foram apenas 12 mil, e também a Meia Maratona Internacional do Rio que foi de 15 mil em 2008 para 20 mil em 2014. A Meia Maratona João Pessoa em 2014 contou com 1200 inscritos, em 2015, esse número chegou a mais de 1500 .

Esses atletas amadores, denominados corredores recreacionais, realizam treinos regulares buscando além da promoção de saúde e melhoria estética, boa classificação na competição ${ }^{5}$. Apesar de realizarem rotinas de treinamento com menores volumes em relação aos atletas profissionais, eles dividem o tempo de treinamento e descanso com suas obrigações cotidianas, familiares, acadêmicas e profissionais.

Outra marcante característica da população de corredores, ainda que amadores, é que os treinos não são apenas moderados, mas, uma combinação com treinamentos intensos, não apenas atividade contínuas, como também intervaladas cuja intensidade pode superar os $100 \%$ do $\mathrm{VO}_{2}$ máximo ${ }^{6}$. Se estas características conferem maior proteção ou se impõe risco cardiometabólico é algo ainda não bem explicado. Por este motivo, este trabalho foi conduzido para avaliar o perfil cardiometabólico de corredores recreacionais e comparar com os valores clínicos considerados protetores cardiometabólicos.

\section{MATERIAL E MÉTODOS}

\section{Sujeitos}

Participaram do estudo 99 atletas corredores recreacionais da cidade de João Pessoa, sendo 69

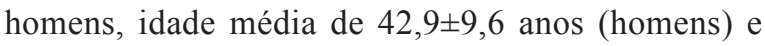
$38,5 \pm 8,4$ anos (mulheres) (Tabela 1). Foram inclusos no estudo aqueles que eram participantes regulares de competições, estavam treinando no mínimo há um ano com frequência semanal de cinco treinos, dos quais no mínimo três eram de corrida, e que não tinham sofrido lesão musculoesquelética tendinosa nos últimos três meses.

\section{Cuidados Éticos}

Os participantes foram esclarecidos quanto aos procedimentos envolvidos e solicitados a assinar o termo de consentimento livre e esclarecido de acordo com a resolução 466/12 do Conselho Nacional de Saúde. A pesquisa foi aprovada pelo Comitê de Ética em Pesquisa do Hospital Universitário Lauro Wanderley, Universidade Federal da Paraíba, sob o protocolo $\mathrm{n}^{\circ}$ $357.246 / 13$. As informações pessoais foram preservadas a fim de evitar possíveis constrangimentos aos voluntários do estudo.

\section{Desenho do Estudo}

Os atletas que consentiram na participação do estudo foram convidados a comparecerem no laboratório sendo orientados a manter um jejum de no mínimo 12 horas. Foi realizada a medida de pressão arterial após 10 minutos de repouso sentados, e uma avaliação antropométrica, após isso, uma coleta sanguínea foi feita para posterior análise do perfil lipídico (colesterol total, Triglicerídeos, HDL-C, LDL-C e VLDLC) perfil glicêmico (glicose), atividades de enzimas hepáticas (Alanina Amino Transferase - ALT e Aspartato Amino Transferase - AST) e função renal (Ácido Úrico, Creatinina e Uréia).

Pressão Arterial: As medidas de pressão arterial (PA) foram tomadas conforme proposto pelas VI Diretrizes Brasileiras de Hipertensão Arterial (2010), a 
partir do método auscultatório utilizando esfigmomanômetro aneróide (Welch Allyn DS4, New York, Estados Unidos). A PA foi verificada três vezes em ambos os braços com intervalo de 5 minutos entre as medidas, e em caso de diferença nas medidas obtidas entre os braços, adotou-se como padrão para o estudo, o braço de maior PA.

Avaliação antropométrica: Avaliou-se massa corporal e estatura (balança antropométrica eletrônica com estadiômetro - Marte, modelo LC 200) para obtenção do Índice de Massa Corporal (IMC). A composição corporal foi avaliada com a balança de bioimpedância elétrica bipolar (Plenna Lumina, MEA02550-Brasil).

Coleta sanguínea: Foram coletados $10 \mathrm{~mL}$ de sangue venoso, retirados da veia antecubital dos voluntários, após jejum de 12 horas por um profissional capacitado. As amostras foram centrifugadas a $3000 \mathrm{rpm}$ por 15 minutos e o sobrenadante (soro ou plasma) transferido para microtubos e refrigerado a $-20^{\circ} \mathrm{C}$ ou $4^{\circ} \mathrm{C}$ até as análises.

Perfil Lipídico: Colesterol Total, Triglicerídeos: As concentrações foram determinadas por meio do kit comercial Colesterol Liquiform (Labtest, Minas Gerais, Brasil), seguindo as instruções do fabricante através do analisador automático Labmax 240 premium.

$H D L-C$ : Os valores foram determinados a partir do kit comercial Labtest (Minas Gerais, Brasil). Um volume de $0,25 \mathrm{~mL}$ de substância precipitante foi adicionado a $0,25 \mathrm{~mL}$ de amostra em tubos e misturados vigorosamente por 30 segundos. Em seguida, este preparo será centrifugado a $3.500 \mathrm{rpm}$ por 15 minutos. Imediatamente após a centrifugação, o sobrenadante límpido foi retirado e colocado em alíquotas contendo 1 $\mathrm{mL}$ do reagente 1 do kit Colesterol Liquiform, e colocado no banho-maria por 10 minutos. Por fim, foi feita a leitura em espectrofotômetro ultravioleta (Biospectro, modelo SP-220/Brasil), a um comprimento de onda de $500 \mathrm{~nm}$, em temperatura ambiente.

$L D L-C$ e $V L D L-C$ : Os valores das lipoproteínas de baixa densidade (LDL-c) e lipoproteínas de muito baixa densidade (VLDL-c) foram estimados pela equação de Friedewald ${ }^{7}[\mathrm{LDL}-\mathrm{C}=(\mathrm{CT}-\mathrm{HDL}-\mathrm{C})-(\mathrm{TG} / 5)]$.

$\mathrm{O}$ índice de Castelli I foi determinado como a razão entre o colesterol total e o HDL-colesterol e o de Castelli II como a razão entre LDL-colesterol e HDLcolesterol $^{8}$.

Perfil Glicêmico: A glicemia de jejum foi determinada em amostras de soro através do método enzimático colorimétrico da glicose oxidase proposto por Trinder ${ }^{9}$, seguindo as recomendações do fabricante, em analisador automático Labmax 240 premium (Labtest, Minas Gerais, Brasil).

Enzimas Hepáticas: As atividades da Alanina Amino Transferase (ALT) e da Aspartato Amino Transferase (AST) foram quantificadas por meio dos kits comerciais ALT/GPT Liquiform eAST/GOTLiquiform (Labtest, Minas Gerais, Brasil), seguindo as instruções do fabricante.

Função Renal: As concentrações de ácido úrico, creatinina e uréia foram quantificadas em soro em analisador automático Labmax 240 por meio dos kits comerciais Ácido Úrico Liquiform, Creatinina K e Ureia UV Liquiform respectivamente, conforme instruções do fabricante (Labtest, Minas Gerais, Brasil).

Análise estatística: Os dados estão apresentados como média \pm desvio padrão da média. Foram aplicados os testes de Komogorov Smirnov e Levene para verificar a normalidade e homogeneidade dos dados, respectivamente. Foi utilizado o teste t para uma amostra e seu correspondente não-paramétrico (teste de Wilcoxon), a fim de observar se as médias estavam significativamente abaixo ou acima dos valores de referência. As análises foram realizadas com o software SPSS Statistics (v. 22, IBM SPSS, Chicago, IL), adotando significância de $\mathrm{p}<0,05$.

\section{RESULTADOS}

$\mathrm{Na}$ tabela 1 estão descritas as características antropométricas gerais e por gênero dos corredores. Homens e mulheres possuíam em média praticamente a mesma faixa etária e Índice de Massa Corporal (IMC), sendo as mulheres detentoras de maior percentual de gordura.

Na tabela 2 estão apresentados os valores dos marcadores glicêmico, lipídico, de pressão arterial e renal de corredores de ambos os sexos juntos. Apenas o indicador lipídico LDLc apresentou-se significativamente elevado em comparação ao valor de referência. $\mathrm{O}$ colesterol total e a uréia chegaram a ficar acima da normalidade, mas apenas descritivamente. Os outros marcadores não excederam os limites de normalidade, caracterizando a amostra geral como normotensa.

Quando estratificado por gênero (Tabela 3), nos homens o marcador lipídico LDLc novamente demonstrou-se significativamente acima do valor de referência. Assim como no resultado de homens e 


\begin{tabular}{|c|c|c|c|}
\hline & Homens & Mulheres & Todos \\
\hline Idade (anos) & $\begin{array}{c}42,9 \pm 9,6 \\
(n=69)\end{array}$ & $\begin{array}{c}38,5 \pm 8,4 \\
(n=30)\end{array}$ & $\begin{array}{c}40,1 \pm 10,5 \\
(n=99)\end{array}$ \\
\hline Massa Corporal (kg) & $\begin{array}{c}70,1 \pm 11,3 \\
(n=69)\end{array}$ & $\begin{array}{c}63,1 \pm 10,2 \\
(n=30)\end{array}$ & $\begin{array}{c}71,0 \pm 12,5 \\
(n=99)\end{array}$ \\
\hline Estatura (cm) & $\begin{array}{c}1,68 \pm 0,0 \\
(n=69)\end{array}$ & $\begin{array}{c}1,61 \pm 0,0 \\
(n=30)\end{array}$ & $\begin{array}{c}1,69 \pm 0,0 \\
(n=99)\end{array}$ \\
\hline IMC (Kg/m²) & $\begin{array}{c}24,6 \pm 2,8 \\
(n=69)\end{array}$ & $\begin{array}{c}24,0 \pm 3,2 \\
(n=30)\end{array}$ & $\begin{array}{c}24,4 \pm 3,0 \\
(n=99)\end{array}$ \\
\hline Gordura (\%) & $\begin{array}{c}17,6 \pm 10,5 \\
(n=66)\end{array}$ & $\begin{array}{c}26,5 \pm 5,0 \\
(n=29)\end{array}$ & $\begin{array}{c}21,2 \pm 15,0 \\
(n=95)\end{array}$ \\
\hline Água (\%) & $\begin{array}{c}15,3 \pm 6,9 \\
(n=68)\end{array}$ & $\begin{array}{c}53,6 \pm 3,6 \\
(n=29)\end{array}$ & $\begin{array}{c}16,5 \pm 7,2 \\
(n=97)\end{array}$ \\
\hline Massa muscular (\%) & $\begin{array}{c}38,9 \pm 4,3 \\
(n=67)\end{array}$ & $\begin{array}{c}33,6 \pm 2,7 \\
(n=29)\end{array}$ & $\begin{array}{c}37,4 \pm 10,9 \\
(n=96)\end{array}$ \\
\hline Massa Óssea (\%) & $\begin{array}{c}10,7 \pm 4,5 \\
(n=68)\end{array}$ & $\begin{array}{c}7,4 \pm 1,1 \\
(n=29)\end{array}$ & $\begin{array}{c}15,3 \pm 13,2 \\
(n=97)\end{array}$ \\
\hline Massa Muscular (Kg) & $\begin{array}{c}28,2 \pm 6,1 \\
(n=68)\end{array}$ & $\begin{array}{c}27,2 \pm 5,4 \\
(n=29)\end{array}$ & $\begin{array}{c}27,7 \pm 5,8 \\
(n=97)\end{array}$ \\
\hline Massa de Gordura (Kg) & $\begin{array}{c}10,7 \pm 5,4 \\
(n=68)\end{array}$ & $\begin{array}{c}14,3 \pm 6,0 \\
(n=29)\end{array}$ & $\begin{array}{c}11,8 \pm 6,1 \\
(n=97)\end{array}$ \\
\hline
\end{tabular}

\begin{tabular}{|c|c|c|c|c|}
\hline & Média \pm DP & $\mathbf{N}$ & $\begin{array}{l}\text { Valor de } \\
\text { referência }\end{array}$ & $\mathbf{P}$ \\
\hline Glicemia (mq/dl) & $94,4 \pm 11,4$ & 99 & 99 & 0,00 \\
\hline Colesterol total (ma/dl) & $202.6 \pm 39.6$ & 99 & 200 & 0.51 \\
\hline Trialicerídeos (ma/dl) & $114.2 \pm 55.7$ & 99 & 150 & 0.00 \\
\hline $\mathrm{HDLc}(\mathrm{mg} / \mathrm{dl})$ & $49,1 \pm 12,3$ & 99 & 60 & 0,00 \\
\hline LDLc (mq/dl) & $130,6 \pm 36,3$ & 99 & 110 & 0,00 \\
\hline VLDLc (ma/dl) & $22,8 \pm 11,1$ & 99 & 40 & 0,00 \\
\hline PAS (mmmHa) & $117,4 \pm 16,3$ & 96 & 139 & 0,00 \\
\hline PAD (mmmHa) & $76.5 \pm 8.7$ & 96 & 89 & 0.00 \\
\hline Uréia (ma/dl) & $45,9 \pm 19,3$ & 99 & 45 & 0,61 \\
\hline \multicolumn{5}{|c|}{$\begin{array}{l}\text { Legenda: HDLc=High Density Lioprotein ; LDLc=Low Density } \\
\text { Liprotein ; VLDLc= Very Low Density Liprotein ; PAS= Pressão } \\
\text { Arterial Sistólica; PAD=Pressão Arterial Diastólica. Valores de } \\
\text { referência: Kit Labtest (Belo Horizonte-MG);VI Diretrizes Brasileiras } \\
\text { da Sociedade Brasileira de Hipertensão10.Os dados estão } \\
\text { apresentados como média } \pm \text { desvio padrão. }\end{array}$} \\
\hline
\end{tabular}




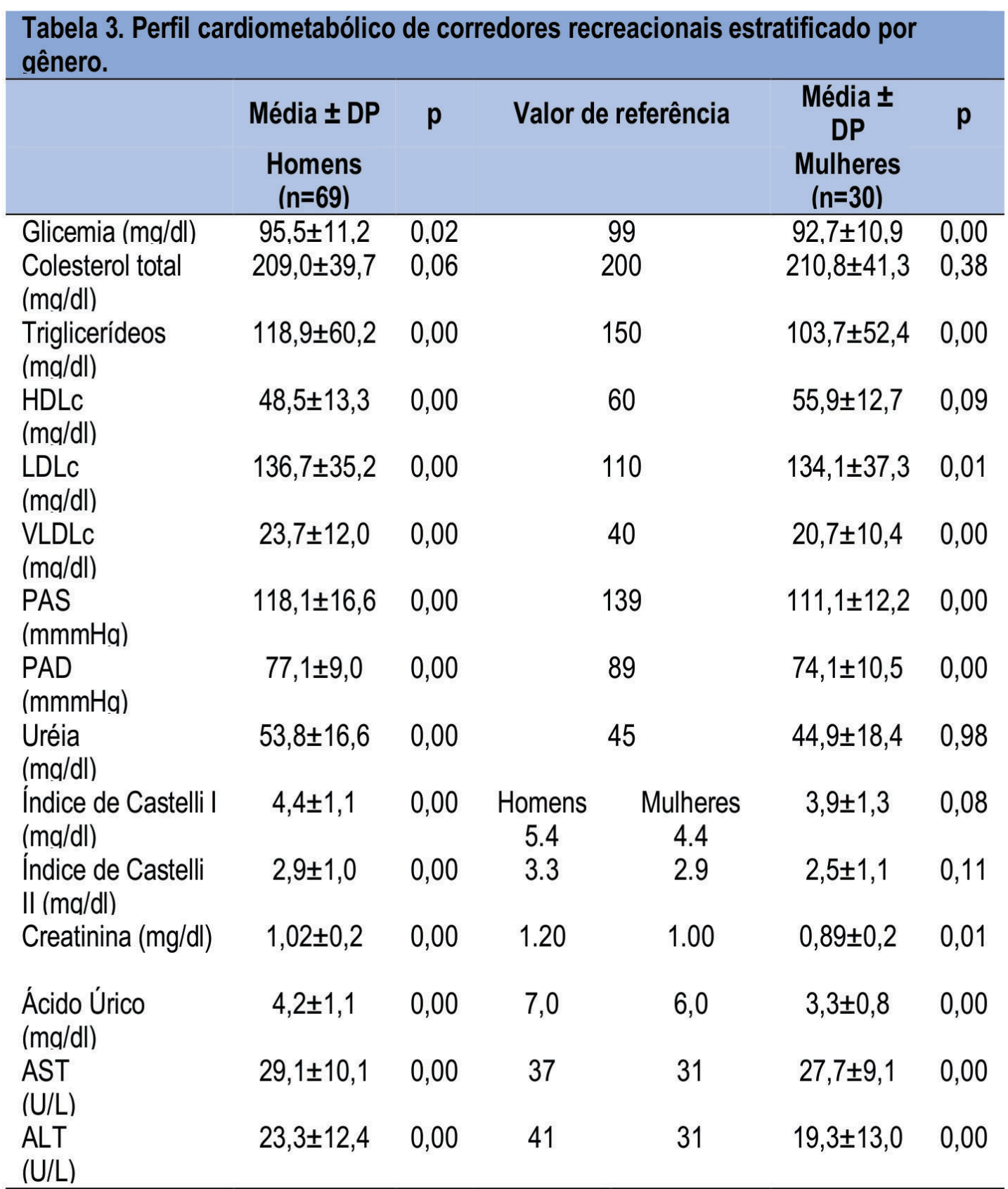

Legenda: HDLc=High Density Lioprotein ; LDLc=Low Density Liprotein ; VLDLc= Very Low Density Liprotein ; AST = Aspartato Amino Transferase; ALT=Alanina Amino Transferase; PAS= Pressão Arterial Sistólica; $\mathrm{PAD}=$ Pressão Arterial Diastólica. Valores de referência: Kit Labtest (Belo Horizonte-MG);VI Diretrizes Brasileiras da Sociedade Brasileira de Hipertensão ${ }^{10}$. Os dados estão apresentados como média \pm desvio padrão. 
mulheres juntos o colesterol total esteve descritivamente acima da normalidade, desta vez apenas para os homens. $\mathrm{O}$ índice de Castelli se manteve dentro da normalidade para ambos os gêneros. Já a uréia encontrou-se significativamente maior que o valor de referência, diferentemente da creatinina que se manteve com valores dentro da normalidade, ambos marcadores da função renal.

O perfil cardiometabólico de corredores recreacionais do gênero feminino também está descrito na tabela 3. Os valores apresentados demonstram que para variáveis de glicemia, triglicerídeos, VLDLc, creatinina, AST e ALT as corredoras encontram-se com níveis significativamente abaixo do limite máximo de referência. Apenas os níveis de LDLc, semelhantemente ao resultado dos corredores recreacionais do gênero masculino, se encontravam em um nível não desejável significativamente acima do limite de referência. Os valores de pressão arterial, bem como os resultados obtidos pelo gênero masculino, também indicavam um nível de classificação de normotensos.

\section{DISCUSSÃO}

Os dados do presente estudo mostraram que os marcadores cardiovasculares, renais e hepáticos da população geral de corredores recreacionais da cidade de João Pessoa encontram-se dentro dos valores de referência para saúde. No entanto, o marcador lipídico LDL se mostrou significativamente acima dos valores de referência no grupo geral e na subdivisão pelo sexo, seguido por níveis também significativamente acima dos valores de referência no colesterol total e uréia no grupo dos corredores do sexo masculino.

Por ser uma modalidade predominantemente aeróbica espera-se que a corrida de rua de forma recreacional promova adaptações fisiológicas em seus praticantes melhorando aspectos da saúde como redução da frequência cardíaca e controle da pressão arterial de repouso que, com o exercício físico, diminui cronicamente a PAS e a PAD, em média, 8,3 mmHg e 5,2 $\mathrm{mmHg}$, respectivamente, estando a redução crônica da PA associada ao efeito cumulativo das reduções $\operatorname{agudas}^{10,11}$, melhoria do perfil lipídico ${ }^{12-14}$, controle do perfil glicêmico ${ }^{15,16}$. De fato, a quantidade de exercício praticado por esses corredores está dentro das recomendações mínimas referidas pelo American College of Sports Medicine para prevenção de doenças cardíacas e hemodinâmicas ${ }^{17}$.

Nesse contexto nossos dados mostraram que a saúde dos corredores se apresenta em bom estado por estarem com os marcadores renais e hepáticos dentro da normalidade, nos quais são capazes de detectar desordens hemodinâmicas nos sujeitos. A creatinina, produto residual da creatina, tem sua transformação no tecido muscular, no qual $1 \%-2 \%$ da creatina livre se converte espontânea e irreversivelmente em creatinina, depende da massa muscular e não apresenta grandes variações diárias ${ }^{18}$. Além disso, alguns outros marcadores avaliados também significativamente abaixo dos valores de referência corroboraram com outro estudo que mostrou menores níveis pressóricos, maiores valores de HDL e menores valores de triglicerídeos de corredores ao comparar-se com os valores de referência ${ }^{19}$. Esses dados mostraram que os corredores apresentaram níveis de triglicerídeos 34\% menores, valores de pressão arterial $2 \%$ maiores e concentração de HDL 14\% menor do que pessoas sedentárias da mesma faixa etária.

Embora esses resultados reflitam uma saúde cardiometabólica satisfatória para os corredores, os valores de LDL encontram-se significativamente acima da referência o que inspira cuidados, pois níveis elevados desse marcador indica a presença da lipoproteína de baixa densidade no plasma que está fortemente associada a episódios vasculares de arteriosclerose, arteriotrombose, acidente vascular encefálico e infarto do miocárdio ${ }^{20,21}$. Essa concentração alterada desse marcador lipídico apresentada pelos corredores do presente estudo diverge com os dados da literatura que mostram baixos níveis de LDL e colesterol total na mesma população ${ }^{22}$. Esse fenômeno pode ser explicado por outros fatores intervenientes que podem ocasionar excesso de LDL no sangue, tais como alimentação e genética ${ }^{23}$. Além disso, corredores recreacionais dividem seu tempo diário entre treinamento e sua vida cotidiana, envolvendo fatores sociais, culturais, relacionamentais entre outros. Nesse caso, os resultados individuais do presente estudo são 
importantes para os sujeitos, de modo que eles podem tomar cuidados necessários para prevenir efeitos deletérios ou procurar assistência médica especializada.

Entretanto o índice de Castelli mostrou-se dentro da normalidade o que pondera os autos níveis de LDL e colesterol total pois eles são compensados pelo HDL, de modo que o colesterol LDL não penetre na parede da artéria pois o HDL faz o transporte reverso do LDL. Desse modo os atletas estão protegidos devido aos altos valores de HDL.

Uma possível causa dos altos valores de uréia apresentados no grupo dos corredores do sexo masculino pode ser o consumo excessivo de aminoácidos normalmente utilizados por atletas que visam a melhoria do desempenho ${ }^{24}$, bem como pode ser o resultado do estresse continuo do treinamento realizado pelos corredores do presente estudo ${ }^{25}$. Portanto é habitual encontrar atletas com valores acima da referência e isso não traz grandes preocupações por ser uma resposta aguda do organismo ${ }^{25}$. No entanto não podemos assegurar esta possibilidade por não avaliarmos o consumo alimentar ou o dano muscular.

A implicação prática desse estudo deve-se ao

\section{REFERÊNCIAS}

1. Sociedade Brasileira de Cardiologia. IV Brazilian Guideline for Dyslipidemia and Atherosclerosis prevention: Department of Atherosclerosis of Brazilian Society of Cardiology. Arq Bras Cardiol. 2007; 88 (Suppl. 1): 2-19.

2. American College of Sports Medicine. ACSM Quantity and Quality of Exercise for Developing and Maintaining Cardiorespiratory, Musculoskeletal, and Neuromotor Fitness in Apparently Healthy Adults: Guidance for Prescribing Exercise. Med Sci Sports Exerc 2011; 43(7): 1334-1359.

3. Brasil. Ministério da Saúde. Vigitel Saúde Suplementar: vigilância de fatores de risco e proteção para doenças crônicas por inquérito telefônico. Brasília, Rio de Janeiro: Agência Nacional de Saúde Suplementar, Ministério da Saúde; 2014.

4. Instituto Ipsos Marplan. Esporte na vida do brasileiro. In: Dossiê Esporte, Um estudo sobre o esporte na vida do brasileiro, $2^{\text {a }}$ Parte; 2006; 61-88.

5. Salgado JVV, Chacon-Mikahil MPT. Corrida de Rua: análise do crescimento do número de provas e de praticantes. Conexões. Revista da Faculdade de Educação Física da UNICAMP. 2006; 4(1): 90-99. fato de trazermos novos marcadores de saúde cardiometabólica ainda não avaliados em corredores, permitindo entender de certa forma os efeitos da prática dessa modalidade. A importância destes dados é que, embora o treinamento físico seja associado com proteção cardiometabólica, a maioria dos estudos é baseada em treinamento de moderada intensidade, enquanto isso, corredores recreacionais costumam realizar sessões de treinamento intervalada e de alta intensidade, cujos efeitos protetores cardiometabólicos são bem menos esclarecidos.

\section{CONCLUSÃO}

Os dados do presente estudo mostraram que o perfil cadiometabólico é considerado bom, uma vez que exceto pelo colesterol LDL, todas as variáveis se encontram dentro dos limites de normalidade mesmo apresentando LDL alto, os altos valores de HDL resultaram em índice de Castelli dentro dos limites de normalidade, indicando que a variável LDL não representa risco aterosclerótico nos atletas.

6. Pazin J, Duarte MFS, Poeta LS, Gomes MA. Recreational road runners: injuries, training, demographics and physical characteristics. Rev Bras Cineantropom Desempenho Hum. 2008;10(3): 277-82.

7. Friedewald WT, Levy RI, Fredrickson DS. Estimation of the concentration of low-density lipoprotein cholesterol in plasma, without use of the preparative ultracentrifuge. Clinical Chemistry, 1972; 18(6): 499-502.

8. Castelli WP. Cholesterol and lipids in the risk of coronary artery disease - the Framingham Heart Study. Can J Cardiol, 1988;4: 5A-10A.

9. Trinder P. Determination of serum cholesterol by enzymatic colorimetric method. Annals of clinical Biochemistry. 1969; (6): 24-27.

10. SBC, SBH, SBN - Sociedade Brasileira de Cardiologia, Sociedade Brasileira de Hipertensão, Sociedade Brasileira de Nefrologia. VI Diretrizes Brasileiras de Hipertensão Arterial. Arq Bras Cardiol. 2010; 1(Supl.1): 1-51.

11. Cornelissen VA, Smart NA. Exercise Training for Blood Pressure: A Systematic Review and Metaanalysis. J Am Heart Assoc. 2013; 1-9. 
12. American College of Sports Medicine. ACSM Exercise and Hypertension. 2004; 533-553.

13. Aadahl M, von Huth Smith L, Pisinger C, Toft UN, Glümer $\mathrm{C}$, Borch-Johnsen $\mathrm{K}$, et al. Five-year change in physical activity is associated with changes in cardiovascular disease risk factors. The Inter99 study. Preventive Medicine. 2009; 48(4): 326-331.

14. Riedl I, Yoshioka M, Nishida Y, Tobina T, Paradis R, Shono N, et al. Regulation of skeletal muscle transcriptome in elderly men after 6weeks of endurance training at lactate threshold intensity. Experimental Gerontology. 2010; 45(11): 896-903.

15. Mann S, Beedie C, Jimenez A. Differential effects of aerobic exercise, resistance training and combined exercise modalities on cholesterol and the lipid profile: review, synthesis and recommendations. Sports Medicine. 2014; 44(2): 211-221.

16. Colberg SR, Sigal RJ, Fernhall B, Regensteiner JG, Blissmer BJ, Rubin RR, et al. Exercise and type 2 diabetes: The American College of Sports Medicine and the American Diabetes Association: Joint position statement. Diabetes Care. 2010; 33(12): 147-167.

17. Sanghani NB, Parchwani DN, Palandurkar KM, Shah AM, Dhanani JV. Impact of lifestyle modification on glycemic control in patients with type 2 diabetes mellitus. Indian Journal of Endocrinology and Metabolism. 2013; 17(6); 1030-9.

18. Pattyn N, Cornelissen VA, Eshghi SRT, Vanhees L. The effect of exercise on the cardiovascular risk factors constituting the metabolic syndrome: A meta-analysis of controlled trials. Sports Medicine. 2013; 43(2): 121133.
19. Taylor BA, Zaleski AL, Capizzi JA, Ballard KD, Troyanos $\mathrm{C}$, Baggish AL, et al. Influence of chronic exercise on carotid atherosclerosis in marathon runners. BMJ Open. 2014; 4(2): 1-6.

20. Williams PT. Walking and running produce similar reductions in cause- specific disease mortality in hypertensives. 2013; 62(3): 485-491.

21. Ridker PM. LDL cholesterol: controversies and future therapeutic directions. Lancet. 2014; 384(9943): 60717.

22. Sodré FL, Costa JCB, Lima, JCC. Avaliação da função e da lesão renal: um desafio laboratorial. J Bras Patol Lab. 2007; 43(5): 329-337.

23. Rubenfire M, Brook RD. HDL cholesterol and cardiovascular outcomes: What is the evidence? Current Cardiology Reports. 2013; 15(4): 1-8.

24. Silva WV, Silva MIAG, Toscano LT, Oliveira KHD, Lacerda LM, SILVA AS. Supplementation prevalence and adverse effects in physical exercise practitioners. Nutrición Hospitalaria. 2014; 29: 158-165.

25. Warburton D, Welsh R, Haykowsky M, Taylor D, Humen D. Biochemical changes as a result of prolonged strenuous exercise. Br. J. Sports Med. 2002; 36(4): 301-303.

\section{Correspondência}

Reabias de Andrade Pereira

Endereço: Rua: Papa João Paulo I

João Pessoa - Paraíba - Brasil - CEP: 58079-808

E-mail: reabiasedf@gmail.com 